\title{
Monitorização não invasiva da pressão intracraniana em pacientes com queixa de cefaleia: série de casos
}

\author{
Luiz Gabriel Gonçalves Cherain ${ }^{1}$ (D), Mateus Gonçalves de Sena Barbosa' (iD, \\ Ghaspar Gomes de Oliveira Alves Francisco ${ }^{1}$ (D) Gustavo Frigieri² ${ }^{\text {ID }}$, Nícollas Nunes Rabelo ${ }^{1}$
}

'Faculdade Atenas, Passos, Minas Gerais, Brasil

2Brain4Care, São Paulo, Brasil

\section{Introdução}

O aumento da pressão intracraniana (PIC) constitui um conjunto de sinais e sintomas relacionados à alteração do volume e da pressão intracraniana, essas alterações são visíveis em diversas doenças neurológicas, o que inclui a cefaleia. Em pacientes com cefaleia crônica a monitorização da pressão intracraniana é realizada de maneira não invasiva o que traz menores danos ao paciente e rapidez nos resultados. O objetivo deste trabalho é elucidar como a monitorização não invasiva da pressão intracraniana em pacientes com cefaleia é realizada e as conclusões a partir da monitorização.

\section{Metodologia}

Estudo de série de casos retrospectiva, onde foram coletados dados clínicos de 32 pacientes atendidos em consultório entre dezembro de 2020 a setembro de 2021, com cefaleia (exceto do tipo enxaqueca), sem restrição de sexo, idade, cor, raça e nível socioeconômico. Todos foram submetidos a monitorização não invasiva da pressão intracraniana por um dispositivo da Brain4care.

\section{Resultados}

Dos 32 pacientes analisados, foi observado que a relação P2/P1 teve seu valor aumentado, com uma média de valores de 1,15 (0,82-1,62). O time to peak teve uma média de 0,23 (0,04-0,34). Esses dados tendem a indicar um aumento da pressão intracraniana e uma diminuição na complacência cerebral em pacientes que relataram queixa de cefaleia. A monitorização não invasiva é importante pois possibilita as medições no consultório, fora do ambiente hospitalar.

\section{Conclusão}

O dispositivo de monitorização não invasiva mostrou uma relação entre o aumento da PIC e a queixa de cefaleia. A vantagem é que a medição pode ser realizada no consultório, utilizando a tecnologia a favor da segurança e conforto do paciente.

Palavras-chave: Complacência intracraniana, Migrânea, Diagnóstico, Tecnologia. 